\title{
Arterial and portal blood supply in cirrhosis: a functional evaluation
}

\author{
P.-M. HUET, A. DU REAU, AND D. MARLEAU \\ From the Clinical Research Center and Department of Medicine, Hôpital Saint-Luc and \\ Université de Montréal, Montreal, Canada
}

SUMMARY The uptake of ${ }^{125 I}$ albumin microaggregates (U-125I-AMA) from portal blood, during a single passage through the hepatic reticuloendothelial system, has been found to be generally decreased in cirrhosis. To investigate if a similar phenomenon occurs for the colloid flowing through the hepatic artery, the U-125I-AMA was first calculated in normal dogs after injection of a mixture of ${ }^{51} \mathrm{Cr}$ red blood cells $\left({ }^{51} \mathrm{Cr}-\mathrm{RBC}\right)$ and ${ }^{125} \mathrm{I}$-AMA into the hepatic artery by comparing hepatic indicator dilution curves (IDC) obtained with both indicators. In nine dogs, the U-125I-AMA from hepatic artery blood was generally over $90 \%$, as previously reported for the same colloid flowing through the portal vein in another group of normal dogs. This approach was then applied in nine patients with alcoholic cirrhosis who underwent combined umbilicoportal vein, hepatic vein, and hepatic artery catheterisation because of severe portal hypertension. Hepatic indicator dilution curves were obtained in the nine patients after injection of a mixture of ${ }^{51} \mathrm{Cr}-\mathrm{RBC}$ and ${ }^{125} \mathrm{I}-\mathrm{AMA}$ into the portal vein and the hepatic artery. The U-125I-AMA from portal and hepatic artery blood was measured by comparing ${ }^{51} \mathrm{Cr}-\mathrm{RBC}$ and ${ }^{125} \mathrm{I}-\mathrm{AMA}$ hepatic IDC. U-125I-AMA varied between $5 \cdot 2$ and $90.5 \%$ after portal vein injection and between 13.7 and $90.1 \%$ after hepatic artery injection; no difference was found between paired values. In all patients the extraction of indocyanine green (E-ICG) was calculated during a continuous infusion and significant correlations were found between E-ICG and U-125I-AMA from portal blood $(r=0.931 ; \mathrm{P}<0.001)$ or from hepatic artery blood $(r=0.861 ; \mathrm{P}<0.005)$. The decreased uptakes can be related to intrahepatic shunts or sinusoidal changes responsible for ineffective phagocytosis and restricted access of dye to parenchymal cells. These data indicate that in cirrhosis the hepatic artery and portal vein blood is cleared of colloid and ICG in a similar fashion and suggest nearly identical blood supply to the regenerative nodules by the hepatic artery and portal vein. Thus U-125I-AMA from hepatic artery or portal vein blood, as well as the E-ICG, may be used to estimate the functional hepatic blood supply in cirrhosis; this may prove to be useful in the prognosis of patients before portacaval shunts.

Cirrhosis has been defined as an altered reconstitution of the lobular parenchyma with both extensive fibrosis and regenerative nodules. In the regenerative nodules, the central vein is displaced eccentrically in the fibrous septa and anastomoses develop with portal veinules (Popper et al., 1952), presumably through the link of persistent sinusoids. We previously studied the Kupffer cell uptake of ${ }^{125} I$ albumin microaggregates flowing through the portal vein and found that, in cirrhosis, the uptake is generally decreased (Huet et al., 1976a). This decreased uptake was related to ineffective phagocytosis (Huet et al., 1976a) secondary to the intra-

Received for publication 21 March 1979 hepatic portohepatic anastomoses and also to sinusoidal changes such as capillarisation (Schaffner and Popper, 1963).

In cirrhosis, although anastomoses between the displaced central veins and hepatic arterioles has been described (Mitra, 1966), it is generally accepted that the regenerative nodules become deprived of their portal blood supply and are vascularised mostly by the hepatic artery (Sherlock, 1975). To investigate the relative importance of the hepatic artery in the functional sinusoidal perfusion, we first studied the Kupffer cell uptake of ${ }^{125}$ I albumin microaggregates (U-125I-AMA) flowing through the hepatic artery in normal dogs. Subsequently, we compared the U-125I-AMA after hepatic artery and portal vein 
injections in patients with alcoholic cirrhosis.

Our data show that, in cirrhosis, there is no difference in the Kupffer cell uptake whether the colloid is injected in the hepatic artery or the portal vein. These findings strongly suggest that, in cirrhosis, the alterations occurring in liver microcirculation affect both hepatic arterioles and portal venules in a similar fashion.

\section{Methods}

\section{NORMAL DOGS}

In a preliminary study, 17 experiments were performed in nine mongrel dogs, each weighing more than $10 \mathrm{~kg}$, anaesthetised with intravenous pentobarbital $(20 \mathrm{mg} / \mathrm{kg})$. In all dogs, a left and a right hepatic vein was catheterised under fluoroscopic visualisation (Huet et al., 1973). The hepatic artery was catheterised through one femoral artery and in all dogs it was verified, using a minimal amount of radiopaque material, that the catheter was not occluding the hepatic artery. The pancreaticoduodenal artery was then ligated.

The indicator mixture contained ${ }^{51} \mathrm{Cr}$ labelled red blood cells $\left.{ }^{51} \mathrm{Cr} \mathrm{RBC}\right)$ and ${ }^{125} \mathrm{I}$ albumin microaggregates ( ${ }^{125}$ I-AMA) (Huet et al., 1976a). After rapid injections of $1 \mathrm{ml}$ of mixture solution into the hepatic artery, the catheter was flushed with saline or blood $(3 \mathrm{ml})$. Samples were collected simultaneously from a right and a left hepatic vein in identical collection racks running at a speed of one tube per second, and analysis of samples was performed as previously described (Huet et al., 1976a). In order to provide a normalisation of the curves and a basis for comparison between indicators, the outflow fractions of injected indicator per $\mathrm{ml}$ of blood were calculated for each tube and were plotted on semilog paper against time (Goresky, 1963). Calculations of indicator dilution curve areas and flows were programmed and computed on a Wang calculator.

Hepatic uptake of ${ }^{125}$ I-AMA: The U-125I-AMA was calculated by comparing hepatic vein indicator dilution curve after injection in the hepatic artery:

$$
\begin{aligned}
& \text { U_-125I-AMA = } \\
& \frac{\mathrm{AUC}\left({ }^{51} \mathrm{Cr} \mathrm{RBC}\right)-\mathrm{AUC}\left({ }^{125} \mathrm{I}-\mathrm{AMA}\right)}{\mathrm{AUC}\left({ }^{51} \mathrm{Cr} \mathrm{RBC}\right)} \times 100
\end{aligned}
$$

in which AUC is the area under the curves obtained with ${ }^{51} \mathrm{Cr}$ RBC and ${ }^{125} \mathrm{I}-\mathrm{AMA}$. This hepatic uptake was expressed as per cent of ${ }^{125}$ I-AMA flowing through the hepatic artery.

In the 17 experiments (nine dogs), the hepatic uptake of ${ }^{125}$ I-AMA and the hepatic blood flow could be estimated simultaneously from one right and one left hepatic vein.

\section{PATIENTS}

Nine patients, eight male and one female, aged 42 to 62 years, who had a haemodynamic evaluation because of bleeding varices secondary to severe portal hypertension (Viallet et al., 1970), were included in this study. All patients had micronodular cirrhosis thought to result from chronic alcoholism; laboratory data and liver biopsy showed no evidence for alcoholic hepatitis and jaundice or ascites were not present. Right hepatic vein catheterisation was performed two days after portal catheterisation under fluoroscopic visualisation (Huet et al., 1976a). In these nine patients, a common hepatic artery could be catheterised by the Seldinger technique. The catheter was advanced beyond the take-off of the gastroduodenal artery and its position was confirmed under fluoroscopic visualisation using minimal amount of radiopaque material. Written informed consent was obtained from each patient.

The indicator mixture injected in the portal vein and in the hepatic artery, in a random order, contained ${ }^{51} \mathrm{Cr} \mathrm{RBC}$ and ${ }^{125} \mathrm{I}$-AMA. In all patients, samples were obtained from one right hepatic vein and collected into a serial collection rack with heparinised tubes (one tube per second). Analysis of samples were performed as previously described for dogs.

\section{HEPATIC EXTRACTION OF ${ }^{125}$ I-AMA}

The U-125I-AMA was calculated by comparing hepatic vein indicator dilution curves after injection in the portal vein and the hepatic artery using the same formula as in dogs. The U-125I-AMA was expressed as percentage of ${ }^{125}$ I-AMA flowing through the portal vein or the hepatic artery (Fig. 1).

In all patients, the hepatic extraction of indocyanine green (E-ICG) and the hepatic blood flow were estimated using a continuous infusion of the dye and sampling from portal vein and one right hepatic vein (Caesar et al., 1961).

Correlation between paired values were tested by Pearson's $r$ test and the mean of individual differences was tested by Student's $t$ test (Snedecor and Cochran, 1967).

\section{Results}

\section{DOGS}

\section{Hepatic uptake of ${ }^{125} I-A M A$}

In the 17 experiments, the mean U-125I-AMA (mean $\pm \mathrm{SE}$ ) was $93.3 \pm 0.8 \%$ (left hepatic vein) and $91 \cdot 8 \pm 1 \cdot 2 \%$ (right hepatic vein) of labelled material flowing through the hepatic artery. The difference between paired values varied between 0 and $11.4 \%$ (mean $3.4 \%$ ) and no significant difference was found $(P>0 \cdot 10)$. U-125I-AMA was constant in the 


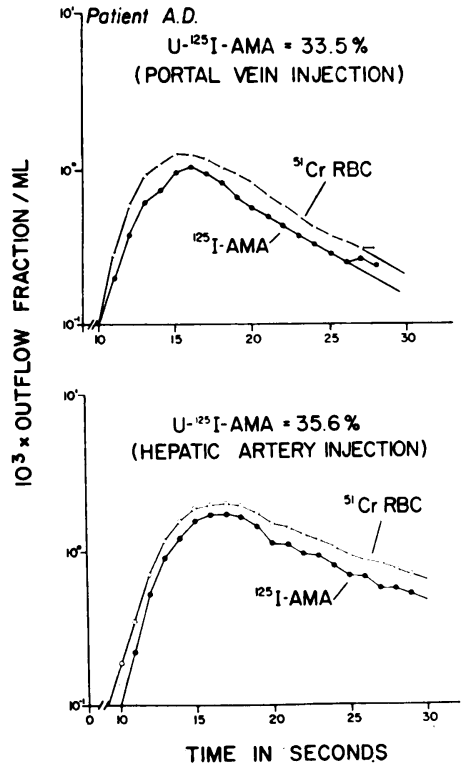

Fig. $1 \quad{ }^{51} \mathrm{Cr}$ labelled red blood cells curves $\left({ }^{51} \mathrm{Cr} \mathrm{RBC}\right)$ and ${ }^{125}$ I albumin microaggregates curves (125I-AMA) obtained from one right hepatic vein after a single injection into the hepatic artery (upper panel) and the portal vein (lower panel) in a cirrhotic patient.

same dogs even after three experiments. These data show that, in normal dogs, the colloid flowing through the hepatic artery was almost completely removed during a single pass through the liver. We previously reported similar values using the same colloid flowing through the portal vein in another group of 15 normal dogs (U-125I-AMA: $92 \cdot 3 \pm 1 \cdot 0 \%$ ) (Huet et al., 1976a).

\section{Hepatic blood flow}

Using the ${ }^{51} \mathrm{Cr} \mathrm{RBC}$ dilution curves, the hepatic blood flow could be estimated simultaneously from a right and a left hepatic vein in the 17 experiments. The mean estimated hepatic blood flows were $1.5 \pm 0.21 / \mathrm{min}$ (left hepatic vein) and $1.6 \pm 0.31 / \mathrm{min}$ (right hepatic vein). However, even though no significant difference was found $(P>0 \cdot 10)$, a wide range of differences existed between paired flows $(r=0 \cdot 187, \mathrm{P}>0 \cdot 5)$.

\section{PATIENTS}

\section{C G studies}

In the nine cases, the hepatic extraction of ICG (E-ICG) varied between 2.3 and $36.6 \%$ (mean $19.2 \%$ ) and the estimated hepatic blood flow between 0.91 and $3.70 \mathrm{l} / \mathrm{min}$ (mean $1.96 \mathrm{l} / \mathrm{min}$ ) (Table).

\section{Indicator dilution studies}

The hepatic uptake of ${ }^{125}$ I-AMA (U-125I-AMA) varied between 13.7 and $90.1 \%$ of colloid flowing through the hepatic artery and between 5.2 and $90.5 \%$ of colloid flowing through the portal vein (Table). No significant difference was found between paired U-125I-AMA $(P>0 \cdot 10)$, which were highly significantly correlated $(r=0.954, \mathrm{P}<0.001)$ (Fig. 2). A highly significant correlation was found between the E-ICG and U-125I-AMA, either after hepatic artery injection $(r=0.861, \mathrm{P}<0.005)$ or after portal vein injection $(r=0.931, \mathrm{P}<0.001)$.

In the nine patients, the hepatic blood flow could be estimated using ${ }^{51} \mathrm{Cr}$ RBC dilution curves after hepatic artery injection and varied between 0.98 and $2.80 \mathrm{l} / \mathrm{min}$ (mean $1.68 \mathrm{l} / \mathrm{min}$ ); however, a wide range of difference existed between flows estimated using this method and ICG method $(r=0.177, \mathrm{P}>0.5)$ (Table). As expected, the estimated hepatic blood flow after portal vein injection was generally

Table Splanchnic hemodynamic data obtained in nine cirrhotic patients

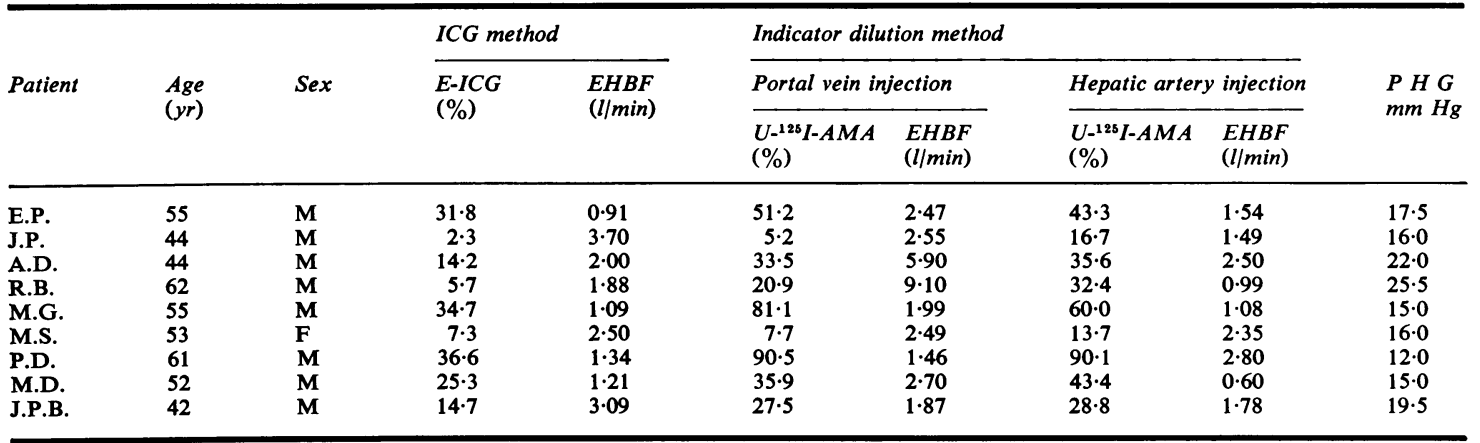

E-ICG: hepatic extraction of Indocyanine green; EHBF: estimated hepatic blood flow; U-125I-AMA: Kupffer cell uptake of ${ }^{125}$ I albumin microaggregates; PHG: portohepatic gradient. 


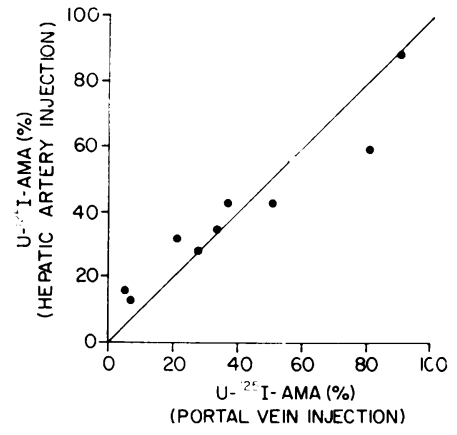

Fig. 2 Comparison of the hepatic uptake of ${ }^{125}$ I albumin microaggregates $(U-125 I-A M A)$ calculated simultaneously after hepatic artery and portal vein injection in nine cirrhotics. The solid line is the line of identity.

overestimated because of loss of indicators through spontaneous portosystemic collaterals (mainly the coronary vein) (Table).

\section{Discussion}

Kupffer cell uptake of ${ }^{125}$ I-AMA from portal blood has been found to be generally over $90 \%$ in normal dogs and in patients with normal or near normal liver (Huet et al., 1976a). In cirrhotic patients, this uptake has been found to be variously decreased and has been proposed as an index of the functional portal blood supply. In the present study, the uptake of ${ }^{125} \mathrm{I}$-AMA from hepatic artery was also found to be over $90 \%$ in normal dogs, values similar to those previously reported for U-125I-AMA from portal blood (Huet et al., 1976a).

In the nine cirrhotic patients, the U-125I-AMA from hepatic artery blood was generally decreased and varied greatly from one patient to another. However, no difference was found when comparing U-125I-AMA flowing through the hepatic artery and the portal vein (Fig. 2). In the nine patients, these uptakes either from portal vein or hepatic artery blood were significantly correlated with the E-ICG.

Our data indicate that hepatic blood flow cannot be accurately estimated from ${ }^{51} \mathrm{Cr}$ curves. The difference between flows estimated simultaneously from one left and one right hepatic vein in dogs and between flows estimated using this method and the ICG method in patients shows that this model does not fulfil all the required conditions for an accurate measurement of hepatic blood flow (Shoemaker et al., 1961; Huet et al., 1973). These data do not confirm those obtained by Cohn et al. (1972).

However, the comparison between ${ }^{51} \mathrm{Cr}$ RBC and ${ }^{125}$ I-AMA dilution curves should be reliable, as both indicators were mixed in the same injected solution and were simultaneously analysed in the hepatic vein samples. This comparison should allow an accurate estimation of the ${ }^{125}$ I-AMA uptake as indicated by the similar uptake calculated repeatedly from right and left hepatic veins in dogs despite the difference in ${ }^{51} \mathrm{Cr}$ RBC dilution curves.

Data from the present study show that hepatic artery and portal vein blood is cleared of colloid and ICG in a similar fashion and do not support a preferential perfusion of regenerative nodule by the hepatic artery (Fig. 3). These findings additionally strengthen the hypothesis that, in cirrhosis, the decreased extraction of tested substances results from a limited access of portal and arterial blood to a relatively normally functioning hepatic cell mass ('intact cell theory') (Shaldon et al., 1961; Branch and Shand, 1976). In chronic liver diseases, the limited access is currently related to intrahepatic shunts or sinusoidal changes such as capillarisation. Several studies using albumin macroaggregates indicate that large communications exist in cirrhosis between the portal vein (Gross et al., 1976; Syrota et al., 1976; Hoefs et al., 1978) or the hepatic artery (Groszmann et al., 1977) and the hepatic veins. Thus, the U-125I-AMA from hepatic artery blood can be used to estimate the functional hepatic artery blood supply and was found to be of the same magnitude as the functional portal blood supply (as evaluated by the U-125I-AMA from portal vein blood) in the nine cirrhotics studied. Consequently, U-125I-AMA from hepatic artery or portal vein blood as well as the E-ICG can be used as indices of the functional

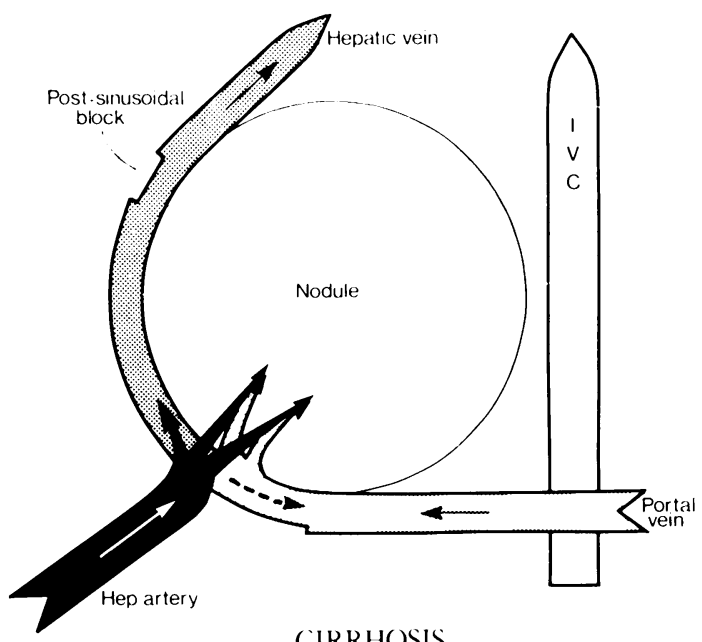

CIRRHOSIS

Fig. 3 The hepatic circulation in cirrhosis. The nodule is supplied by the hepatic artery and portal vein in a similar fashion (modified from Sherlock, 1968) 
hepatic blood supply in cirrhosis, or the blood mass effectively in contact with hepatic and Kupffer cells allowing the uptake process.

Inability to predict in advance which patients will do poorly after portacaval shunts has limited the enthusiasm in surgical treatments as well as in splanchnic haemodynamic studies (Reynolds, 1974). However, the preoperative estimation of the functional hepatic blood supply may prove to be useful in predicting the postoperative course. The complex approach of the present study (U-125I-AMA) validates the estimation of this parameter by a relatively simple technique (E-ICG) which can be available in most hospitals. We have recently reported preliminary data suggesting that the occurrence of portosystemic encephalopathy and/or progressive liver failure after portacaval shunt could be related in cirrhotics to the preoperative E-ICG used as an index of the functional hepatic blood supply (Huet et al., 1976b).

We thank Dr A. Viallet for his criticism in reviewing the manuscript and Dr M. Lafortune for his help in hepatic artery catheterisation. We also thank M. J. Lachance, J. Pruneau, and J. Marchi for illustration and F. Trotier for secretarial assistance. This work was supported by the Medical Research Council of Canada (PG-3).

\section{References}

Branch, R. A., and Shand, D. G. (1976). Propranolol disposition in chronic liver disease: a physiological approach. Clinical Pharmacokinetics, 1, 264-279.

Caesar, J., Shaldon, S., Chiandussi, L., Guevara, L., and Sherlock, S. (1961). The use of Indocyanine green in the measurement of hepatic blood flow and as a test of hepatic function. Clinical Sciences, 21, 43-57.

Cohn, J. N., Khatri, I. M., Groszmann, R. J., and Kotelanski, B. (1972). Hepatic blood flow in alcoholic liver disease measured by an indicator dilution technic. American Journal of Medicine, 53, 704-714.

Goresky, C. A. (1963). A linear method for determining liver sinusoidal and extravascular volumes. American Journal of Physiology, 204, 626-640.

Gross, G., Babel, J. F., Ritschard, J., Megevand, R., Rohner, A., Donath, A., and Perrier, C. V. (1976). Quantification of intrahepatic portasystemic shunting in cirrhotic patients: possible relevance to the problem of indication for surgical procedure. In The Liver. Quantitative Aspects of Structure and Function, pp. 159-166. Edited by R.
Preisig, J. Bircher, and C. Paumgartner. Editio Cantor; Aulendorf.

Groszmann, R. J., Kravetz, D., and Parysow, O. (1977). Intrahepatic arteriovenous shunting in cirrhosis of the liver. Gastroenterology, 73, 201-204.

Hoefs, J., Sakimura, I.. and Reynolds. T. (1978). Direct measurement of intrahepatic shunting by the portal vein injection of microspheres (Abstract). Gastroenterology, 75, 968.

Huet, P. M., Lavoie, P., and Viallet, A. (1973). Simultaneous estimation of hepatic and portal blood flows by an indicator dilution technique. Journal of Laboratory and Clinical Medicine, 82, 836-846.

Huet, P. M., Marleau, D., Lavoie, P., and Viallet, A. (1976a). Extraction of ${ }^{125} \mathrm{I}$-albumin microaggregates from portal blood: an index of functional portal blood supply in cirrhotics. Gastroenterology, 70, 74-81.

Huet, P. M., Marleau, D., Lavoie, P., Lim, E., Tanguay, S., and Viallet, A. (1976b). 'Functional' portal blood supply (PBS): a useful criteria in prognosis and selection for portacaval shunt in cirrhotics (Abstract). Gastroenterology, 71, 912.

Mitra, S. K. (1966). Hepatic vascular changes in human and experimental cirrhosis. Journal of Pathology and Bacteriology, 92, 405-414.

Popper, H., Elias, H., and Petty, D. E. (1952). Vascular pattern of the cirrhotic liver. American Journal of Clinical Pathology, 22, 717-729.

Reynolds, T. B. (1974). Promises! promises! Hemodynamics and portal-systemic shunt. New England Journal of Medicine, 290, 1484-1485.

Schaffner, F., and Popper, H. (1963). Capillarization of hepatic sinusoids in man. Gastroenterology, 44, 239-242.

Shaldon, S., Chiandussi, L., Guevara, L., Caesar, J., and Sherlock, S. (1961). The estimation of hepatic blood flow and intrahepatic shunted blood flow by colloidal heatdenatured human serum albumin labeled with $\mathrm{I}^{131}$. Journal of Clinical Investigation, 40, 1346-1354.

Sherlock, S. (1968). The portal venous system and portal hypertension. In Diseases of the Liver and Biliary System, pp. 163-236. 4th ed. Blackwell: Oxford.

Sherlock, S. (1975). The portal venous system and portal hypertension. In Diseases of the Liver and Biliary System, pp. 150-216. 5th ed. Blackwell: Oxford.

Shoemaker, W. C., Steenburg, R. W., Smith, L. L., and Moore, F. D. (1961). Experimental evaluation of an indicator-dilution technique for estimation of hepatic blood flow. Journal of Laboratory and Clinical Medicine, 57, 661-670.

Snedecor, G. W., and Cochran, W. G. (1967). Statistical Methods, pp. 91-119. 6th ed. Iowa State University Press: Ames, Iowa.

Syrota, A., Vinot, J. M., Paraf, A., and Roucayrol, J. C. (1976). Scintillation splenoportography: hemodynamic and morphological study of the portal circulation. Gastroenterology, 71, 652-659.

Viallet, A., Legare, A., and Lavoie, P. (1970). Hepatic and umbilicoportal catheterization in portal hypertension. Annals of the New York Academy of Sciences, 170, 177-192. 